\title{
Control of Italian ryegrass and Alexandergrass in corn using different corn sowing date, pre- and post-emergent herbicides
}

\author{
Cristiano Piasecki1 ${ }^{1, \star}$ (D), Ramiro Fernando Lopez Ovejero² (D), Gilmar José Picoli Junior² (iD, Luiz \\ Francisco Bellini ${ }^{\text {(D) }}$, Dirceu Agostinetto ${ }^{1}$ (D), Leandro Vargas ${ }^{3}$ (D) \\ 1. Universidade Federal de Pelotas - Departamento de Fitossanidade - Centro de Herbologia - Capão do Leão (RS), Brazil. \\ 2. Bayer Cropscience Brasil - São Paulo (SP), Brazil. \\ 3. Empresa Brasileira de Pesquisa Agropecuária - Weed Science Department - Passo Fundo (RS), Brazil.
}

\begin{abstract}
Glyphosate-resistant (GR) Italian ryegrass (LOLMU) and Alexandergrass (URPLA) are troublesome weeds in corn cropping systems in Southern Brazil. The emergence pattern of those weeds is not uniform and may change according to the season's environmental characteristics. Also, herbicide resistance has been diminishing the success of the weed control programs. The objectives of this study were to evaluate the influence of corn-sowing date on LOLMU and URPLA densities and their control provided by pre- and post-emergent herbicides. Field trials were conducted in two crop seasons in Southern Brazil consisting of three corn sowing date (August, September, and October) and the application of atrazine + S-metolachlor (residual) in corn pre-emergence in different post-emergence weed control programs with glyphosate, ammonium-glufosinate, nicosulfuron, and atrazine. The results indicated that the sowing date had a significant influence on LOLMU and URPLA densities. Corn sown in the earliest period was exposed to a higher LOLMU density, whereas corn sown in the latest period had a higher density of URPLA. Also, the application of residual herbicide at corn pre-emergence reduced both weed species densities and decreased the pressure for the control of glyphosate-resistant LOLMU for the post-emergence herbicides. The use of residual herbicides in corn pre-emergence is an efficient strategy to be considered in the LOLMU and URPLA control programs, followed by post-emergence application of glyphosate, ammonium-glufosinate, atrazine, and nicosulfuron.
\end{abstract}

Key words: atrazine, integrated weed management, herbicide resistance management, S-metolachlor, residual.
Received:

Sep. 14, 2019

Accepted:

May. 21, 2020

Section Editor:

Gabriel Constantino Blain

${ }^{*}$ Corresponding author: c piasecki@hotmail.com

\section{INTRODUCTION}

Corn is an important cash crop in the Rio Grande do Sul state, Brazil. It is cultivated in an area of approximately 1 million hectares and produces nearly 6,000 tons per year (CONAB 2018). In this state, the significant part of the corn sowing window ranges from August to October, but it can be stretched out until December in some regions. In this case, corn is prone to suffer interference of weeds established during fall/winter, such as glyphosate-resistant (GR) Italian ryegrass (Lolium multiflorum L.) (LOLMU) (Nandula 2014) and Alexandergrass [Urochloa plantaginea (Link) R.D. Webster] (URPLA), formerly named Brachiaria plantaginea (Link) (BRAPL), during end-winter/spring (Galon et al. 2008).

Italian ryegrass is $\mathrm{a}_{3}$ annual grass whose reproduction occurs by seeds, which can germinate, emerge, and establish over a range of environmental conditions (Bond et al. 2014; Roman et al. 2004). This species grows up vigorously and is highly 
competitive (Bond et al. 2014). Also, in Southern Brazil, LOLMU is typically cultivated as pasture or cover crop prior to corn sowing, which favors its establishment in high density (Roman et al. 2004). High density and not adequate stage of GR LOLMU are two factors that make it difficult to control with a burndown application before corn establishment. The poorly controlled plants will likely interfere with corn development and can reduce the crop yield up to 48\% (Nandula 2014; Fipke and Vidal 2019).

Alexandergrass is $\mathrm{a}_{4}$ annual grass that reproduces by seeds (Khatounian et al. 2016). URPLA is well adapted in several tropical and subtropical agroecosystems (Velho et al. 2012; Macedo et al. 2019). In Brazil, URPLA is endemic in most agricultural regions, infesting several crops, from grains to vegetables and orchards (Khatounian et al. 2016); it is the most common weed grass in corn and can reduce the crop yield by up to 80\% (Galon et al. 2008; Kozlowski 2002).

The control of LOLMU and URPLA in Brazilian commercial corn fields have been performed in the past decade with herbicide applications, especially glyphosate (5-enolpyruvylshikimate 3-phosphate synthase - EPSPS inhibitor) (Galon et al. 2008; Vargas et al. 2005). However, widespread glyphosate applications have selected GR LOLMU populations (Heap 2020). The first case of GR LOLMU in Rio Grande do Sul was reported in 2003 (Roman et al. 2004). Recently, GR LOLMU was reported on about $85 \%$ of the state's 4 million hectares of agricultural area (Vargas et al. 2016). In contrast, URPLA is wide-distributed in the crop fields in Rio Grande do Sul, being considered companion weed to corn (Galon et al. 2008). Currently, there are no reports of GR URPLA in Brazil, however, there is cases involving resistance to acetyl coenzyme-A carboxylase (ACCase) inhibitors in another state (Heap 2020).

Proactive actions are required to avoid or delay the evolution of URPLA caused by the repetitive application of glyphosate. In the context of weed evolution to herbicide resistance, the employment of integrated weed management practices (IWM) is essential. The association of cultural practices (sowing date, crop rotation, etc.) with pre- and post-emergence herbicides are strategies that should be implemented in order to provide sustainability to the agricultural system in Rio Grande do Sul and consequently in Brazil.

The control of GR LOLMU and URPLA in Rio Grande do Sul is generally performed with a burndown application of glyphosate tank-mixed with ACCase inhibitors at 21-30 days before sowing, followed by a preplant application of photosystem I and/or II inhibitors (PSI, PSII) 1-3 days before sowing. In corn post-emergence, the most applied herbicides programs by farmers to control those weeds are glyphosate, ammonium-glufosinate (glutamine synthetase - GS inhibitor) in tolerant-corn hybrids, nicosulfuron (acetolactate synthase inhibitors - ALS), and mesotrione and/or tembotrione (4-hydroxyphenylpyruvate dioxygenase inhibitors - HPPD inhibitors), usually tank-mixed with atrazine.

The current struggles in controlling LOLMU and URPLA in corn post-emergence indicates the need for a more efficient burndown and preemergent herbicide program. The emergence pattern of LOLMU and URPLA may vary with seasons depending upon environmental conditions; however, in the corn fields of Rio Grande do Sul, this variation is still poorly understood. In this way, the hypothesis of the present study were that corn sown early in the growing season is exposed to more significant interference from LOLMU. Differently, corn sown at the end of the growing season is subject to more significant interference from URPLA. In addition, residual herbicide applied at corn pre-emergence can improve the control of both LOLMU and URPLA post-emergence. Therefore, the objectives of this work were to evaluate the influence of corn-sowing date on LOLMU and URPLA densities and their control provided by residual herbicide treatment in corn pre-emergence followed by post-emergence herbicides programs.

\section{MATERIAL AND METHODS}

\section{Field experiments description and treatments}

Two field trials were conducted at Bayer Research Station, located at Não-Me-Toque, Rio Grande do Sul state (32³8'17' S, $68^{\circ} 55^{\prime} 74^{\prime \prime} \mathrm{W}$ at $514 \mathrm{~m}$ of altitude), during the 2014/2015 and 2015/2016 growing seasons. The soil where the trials were sown was a dystrophic red latosol (Embrapa 1999), with an average of $49 \%$ of clay, $29 \mathrm{~g} \cdot \mathrm{dm}^{3}$ of organic matter content, and $\mathrm{pH}$ (water) of 5.6. Both experiments were performed in an area infested with GR LOLMU and glyphosate-sensitive URPLA. Glyphosateresistance status of both weed species was previously confirmed by whole-plant dose-response studies (data not shown). 
In each growing season, the corn sowing was performed three distinct dates with an interval of about 20 days between operations (Table 1). The sum of rainfall events observed at the research station during the experimental period was $1790 \mathrm{~mm}$ (season 2014/2015: from 08/01/2014 to 05/01/2015) and $1605 \mathrm{~mm}$ (season 2015/2016: from 09/01/2015 to 06/30/2016). Temperature is shown in Fig. 1. According to each sowing date, weeds were previously controlled with glyphosate at $1,440 \mathrm{~g}$ ae $\cdot \mathrm{ha}^{-1}$ (Roundup WG, Monsanto) plus clethodim at $72 \mathrm{~g}$ ai $\cdot \mathrm{ha}^{-1}$ (Select, Arysta LifeScience) 30 days before sowing, followed by paraquat at $400 \mathrm{~g}$ ai $\cdot \mathrm{ha}^{-1}$ plus diuron at $200 \mathrm{~g}$ ai $\cdot \mathrm{ha}^{-1}$ (Gramocil, Syngenta Crop Protection) two days before sowing (Table 2 ).

The corn hybrids used were glyphosate- and ammonium-glufosinate tolerant (2014/2015 - 2A620 PW; and 2015/2016 30A77 PW). The seeds for all three sowing dates were mechanically sown in a no-till system with a planting depth of 3 to $5 \mathrm{~cm}$ and a seeding rate of 4 plants $\cdot \mathrm{m}^{-1}$ (Table 1). Plots were $5 \mathrm{~m}$ long with five rows spaced $50 \mathrm{~cm}$ apart $\left(12.5 \mathrm{~m}^{2}\right)$. Fertilizer rate was in accordance with the recommendations of soil analysis, and consistent with the local grower practice of $32 \mathrm{~kg}$ of $\mathrm{N}$, $112 \mathrm{~kg}$ of $\mathrm{P}_{2} \mathrm{O}_{5}, 80 \mathrm{~kg}$ of $\mathrm{K}_{2} \mathrm{O} \cdot \mathrm{ha}^{-1}$ in-furrow at sowing. At the $\mathrm{V}_{6-} \mathrm{V}_{7}$ corn leaf stage, $184 \mathrm{~kg} \mathrm{~N} \cdot \mathrm{ha}^{-1}$ (urea) was broadcast applied.

All herbicides treatments were applied using a pressurized backpack sprayer, equipped with four spray nozzles (Teejet TT110015), spaced $50 \mathrm{~cm}$ apart, calibrated to deliver $120 \mathrm{~L} \cdot \mathrm{ha}^{-1}$. The residual herbicides (PRE) were applied at the day of corn sowing, whereas the post-emergent herbicides (POST) application were at the $\mathrm{V}_{4}-\mathrm{V}_{5}$ stage (4 to 5-leaf stage). Detailed information on herbicides usage as well as dose and timing of treatment in PRE and POST in both growing seasons are presented in Tables 1-5.

\section{Experimental design, evaluated variables, and statistical analyses}

The experiments were performed in a randomized block design with four replicates arranged in a two-way factorial $3 \times 9$. The first factor consisted of three corn sowing dates and the second factor of nine herbicide programs in PRE and POST (Tables 3-5).

The evaluated variables were weed density (LOLMU and URPLA - plants $\cdot \mathrm{m}^{-2}$ ) and corn yield. The weed density was assessed 28 days after preemergent herbicides application (28 DAPRE $-V_{4}-V_{5}$ corn leaf stage) and 21 days after post-emergent herbicides application (21 DAPOST). In each evaluation, the weed density was twice measured per plot with a $0.25-\mathrm{m}^{2}$ square frame randomly placed, and the number of plants $\mathrm{m}^{-2}$ calculated. The corn yield was determined by the manual harvest of the two central rows in each experimental unit. Corn kernels were threshed, grain moisture and weight measured, and, after adjusting the moist to $13 \%$, the corn yield $\mathrm{kg} \cdot \mathrm{ha}^{-1}$ was calculated.

The results higher or lower than 50\% of the upper and lower limits (quartiles Q3 and Q1) were considered outliers and removed. The data were checked for normality by Shapiro-Wilk's test and after subjected to ANOVA at F test $\mathrm{p} \leq 0.05$. Interactions between factors were verified at $\mathrm{p} \leq 0.05$, and characters that presented significant interaction split to identify the qualitative (sowing dates) and quantitative (herbicides) simple effects of each treatment on evaluated variables. Tukey's test was used to compare means at $\mathrm{p} \leq 0.05$. A joint analysis was used to assess the impact of the year of the experiment on the evaluated variables, according to Banzatto and Kronka (2008). The statistical program CoStat package was used for statistical analysis (Cardinali and Nason 2013).

Table 1. Sowing and application dates of the corn in cropping season 2014/2015 and 2015/2016.

\begin{tabular}{cccc}
\hline \multirow{2}{*}{ Crop Season } & \multicolumn{3}{c}{ Sowing and herbicide treatment in PRE ${ }^{\mathrm{a}}$} \\
\cline { 2 - 4 } & $\mathbf{1}$ & $\mathbf{2}$ & $\mathbf{3}$ \\
\hline $2014 / 2015$ & $08 / 22 / 2014$ & $09 / 10 / 2014$ & $10 / 01 / 2014$ \\
\hline $2015 / 2016$ & $09 / 23 / 2015$ & $10 / 13 / 2015$ & $10 / 28 / 2015$ \\
\hline \multirow{2}{*}{ Crop Season } & Date of herbicide treatment in POST according to each Sowing date \\
\hline $2014 / 2015$ & $\mathbf{1}$ & $\mathbf{2}$ & $\mathbf{3}$ \\
\hline $2015 / 2016$ & $9 / 26 / 2014$ & $10 / 18 / 2014$ & $11 / 4 / 2014$ \\
\hline
\end{tabular}

${ }^{a}$ Abbreviation: PRE, treatment performed in corn pre-emergence on the same day of sowing according to each time. POST, treatment performed in corn postemergence at $\mathrm{V}_{4}-\mathrm{V}_{5}$ stage ( 4 to 5-leaf stage). 

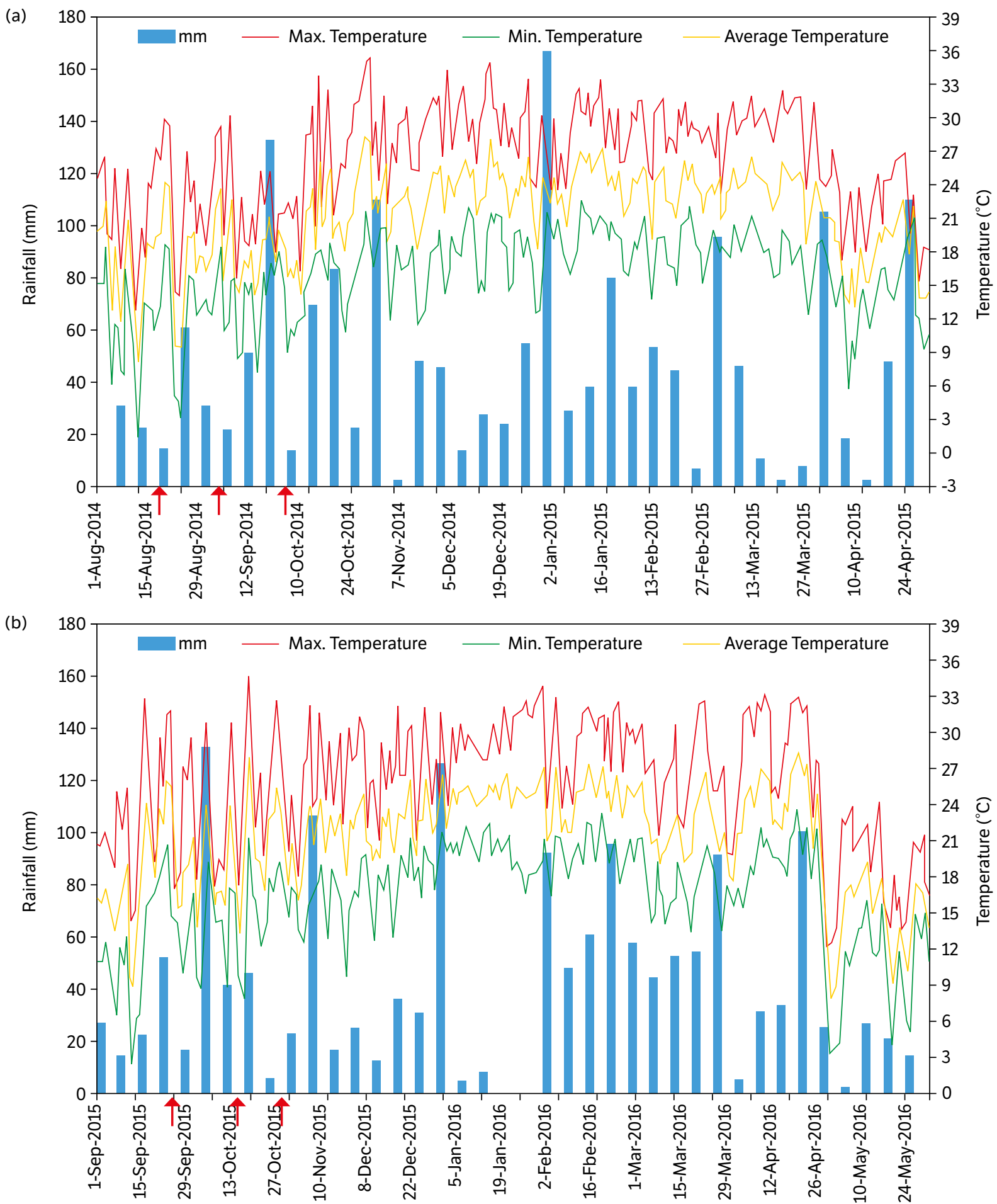

Figure 1. Weekly rainfall (mm) and air temperature $\left({ }^{\circ} \mathrm{C}\right.$ ) in (a) 2014/2015 and (b) 2015/2016 cropping season during the experimental period in Não-Me-Toque, Rio Grande do Sul state, Brazil. Weather data obtained from an automated meteorology station. Red arrows indicate the sowing date 1,2 , and 3 respectively in each cropping season.

\section{RESULTS AND DISCUSSION}

A total of $7.2 \%$ of the weed density data was considered outliers and removed from the original results, whereas there were no outliers for corn grain yield. The data presented normal distribution without the need for transformation. The joint 
Table 2. Herbicide common and trade names, and herbicide manufacturer information for herbicides used in both experiments in a 2014/2015 and 2015/2016 cropping season.

\begin{tabular}{ccc}
\hline Common name & Trade name $^{1}$ & Manufacturer \\
\hline Atrazine + S-metolachlor & & \\
\hline Atrazine & Primestra & Syngenta Crop Protection, São Paulo, SP, Brazil \\
\hline Glyphosate & Gesaprim & Syngenta Crop Protection, São Paulo, SP, Brazil \\
\hline Ammonium-glufosinate & Roundup Ready Milho & Monsanto do Brasil, São Paulo, SP, Brazil \\
\hline Nicosulfuron & Finale & Bayer CropScience, Michigan, Ml, United States \\
\hline Clethodim & Sanson & Arysta Lifescience, Salto de Pirapora, SP, Brazil \\
\hline Glyphosate & Select & Arysta Lifescience, Salto de Pirapora, SP, Brazil \\
\hline Paraquat + Diuron & Roundup WG & Monsanto do Brasil, São Paulo, SP, Brazil \\
\hline
\end{tabular}

${ }^{1}$ Adjuvants were added for each herbicide treatment according to manufacturer recommendations. ${ }^{2}$ Pre-mixed formulations.

analysis showed significant differences between growing seasons, and, then, the data was separately analyzed. The ANOVA showed a significant difference for the factor level's (sowing dates and herbicides) for the evaluated variables except for URPLA density at 21 POST in the 2015/2016 season. Significant interactions were observed only for the LOLMU density evaluated at 28 DAPRE in both the 2014/2015 and 2015/2016 crop seasons.

The variability of the results observed was considered high, which is expected once the natural weed distribution along the ground surface is not uniform (Radosevich et al. 2007). The variation reduced the utilization of collected data (about $7.2 \%$ of outliers) and also favored a higher coefficient of variation of statistical results (Tables 3-5).

During the 2015/2016 season, a drought period delayed the installation of the experiment in 32 days (Table 1, Fig. 1). This delay likely influenced the results due to the difference in environmental conditions during the establishment of the second-year experiment, resulting in significant effects between the two years of the study. Although this difference between growing seasons did not allow the data to be pooled, it did increase the period of establishment of this study, which is from Aug 22th to Oct 28th (Table 1).

\section{Effects of corn sowing date on LOLMU and URPLA densities}

The results of the sowing dates on weed density can be better observed in the treatments when residual herbicides (atrazine + S-metolachlor) were not applied because it represents the original weed infestation (Tables 3 and 4 ). The sowing date influenced the LOLMU and URPLA densities in both the 2014/2015 and 2015/2016 crop seasons (Tables 3 and 4). The two-earliest sowing dates had the highest LOLMU density in both seasons, comprising from Aug 22th to Oct 13th (Tables 1 and 3). LOLMU is a winter/spring plant, and the germination of its seeds is influenced by temperature, light, storage environment, and age (Gramshaw 1972).

In contrast, the highest URPLA density occurred in the last sowing date during the 2014/2015 season and the first sowing date in 2015/2016, which is a similar time of the year in both seasons (from Sep 23th to Oct 1st) (Tables 1 and 4). URPLA germinates in spring/summer conditions and achieves its maximum vigor under the hot and wet season of typical tropical climates (Khatounian et al. 2016).

Temperature affects the weedy-seed germination (Radosevich et al. 2007; Khatounian et al. 2016). In the present study, lower temperatures favored LOLMU's germination, while warmer conditions benefit URPLA (Fig. 1). This data showed that the initial corn sowing dates (August and September) had lower temperatures than the third sowing date (as expected for the reason of the time of the year). The average temperatures from corn sowing day until 28 days after emergence was $17.7,17.9$, and $21^{\circ} \mathrm{C}$ in the $2014 / 2015$, whereas $19.4,20.6$, and $21.5^{\circ} \mathrm{C}$ during $2015 / 2016$, respectively for sowing dates 1,2 , and 3 (Fig. 1). Also, it is possible to observe that due to the drought period registered in the 2015/2016 season, the general weed density was lower than the previous season 2014/2015 (Tables 3 and 4). 
Table 3. The density of Lolium multiflorum (LOLMU) (plants $\mathrm{m}^{-2}$ ) at 28 days after treatment with preemergent herbicides (28 DAPRE) and 21 days after treatment with post-emergent herbicides (21 DAPOST) evaluated in three sowing date s and two cropping seasons $2014 / 2015$ and $2015 / 2016$.

\begin{tabular}{|c|c|c|c|c|c|c|c|c|c|}
\hline \multicolumn{10}{|c|}{ Season $2014-2015^{c}$} \\
\hline \multirow{3}{*}{$\begin{array}{c}\text { Treatments } \\
\left.\text { PRE (g ai } \cdot \mathrm{ha}^{-1}\right)\end{array}$} & \multicolumn{4}{|c|}{ Plants $\mathrm{m}^{-2}$ at 28 DAPRE $^{\mathrm{a}}$} & \multirow{3}{*}{$\begin{array}{c}\text { Treatments } \\
\text { POST (g ai or ae } \cdot \mathrm{ha}^{-1} \text { ) }\end{array}$} & \multicolumn{4}{|c|}{ Plants $\mathrm{m}^{-2}$ at 21 DAPOST $^{\mathrm{b}}$} \\
\hline & \multicolumn{3}{|c|}{ Sowing time } & \multirow[t]{2}{*}{ Mean } & & \multicolumn{3}{|c|}{ Sowing time } & \multirow[t]{2}{*}{ Mean } \\
\hline & 1 & 2 & 3 & & & 1 & 2 & 3 & \\
\hline $\begin{array}{c}\text { Atrazine }(1,480)+ \\
\text { S-metolachlor }(1,160)\end{array}$ & BC $36 \mathrm{a}$ & B $23 a b$ & $\mathrm{BOC}$ & - & Untreated & 14 & 4 & 1 & $\mathrm{BC} 6$ \\
\hline $\begin{array}{c}\text { Atrazine }(1,480)+ \\
\text { S-metolachlor }(1,160)\end{array}$ & $\mathrm{C} 23 \mathrm{a}$ & B $10 a b$ & $\mathrm{~B} 1 \mathrm{~b}$ & - & Atrazine $(1,500)$ & 10 & 1 & 0 & $\mathrm{BC} 4$ \\
\hline $\begin{array}{c}\text { Atrazine }(1,480)+ \\
\text { S-metolachlor }(1,160)\end{array}$ & BC 27 a & B $12 a b$ & $\mathrm{~B} 1 \mathrm{~b}$ & - & $\begin{array}{l}\text { Atrazine }(1,500)+ \\
\text { glyphosate }(720)\end{array}$ & 7 & 3 & 0 & C3 \\
\hline $\begin{array}{c}\text { Atrazine }(1,480)+ \\
\text { S-metolachlor }(1,160)\end{array}$ & C15a & B 14 a & $\mathrm{B} 1 \mathrm{~b}$ & - & $\begin{array}{l}\text { Atrazine }(1,500)+\text { ammonium- } \\
\text { glufosinate }(500)\end{array}$ & 5 & 2 & 0 & $\mathrm{C} 2$ \\
\hline $\begin{array}{c}\text { Atrazine }(1,480)+ \\
\text { S-metolachlor }(1,160)\end{array}$ & BC 27 a & B $12 b$ & $\mathrm{~B} 1 \mathrm{C}$ & - & $\begin{array}{l}\text { Atrazine }(1,500)+ \\
\text { nicosulfuron }(32)\end{array}$ & 6 & 2 & 0 & $\mathrm{C} 2$ \\
\hline Untreated & A 98 a & A $122 \mathrm{a}$ & A $13 b$ & - & $\begin{array}{l}\text { Atrazine }(1,500)+ \\
\text { glyphosate }(720)\end{array}$ & 35 & 29 & 6 & A 23 \\
\hline Untreated & A $87 b$ & A $156 a$ & A $11 \mathrm{c}$ & - & $\begin{array}{c}\text { Atrazine }(1,500)+\text { ammonium- } \\
\text { glufosinate }(500)\end{array}$ & 15 & 1 & 1 & $\mathrm{BC} 5$ \\
\hline Untreated & A 92 b & A 150 a & $A B 9 c$ & - & $\begin{array}{l}\text { Atrazine }(1,500)+ \\
\text { nicosulfuron }(32)\end{array}$ & 19 & 2 & 1 & $\mathrm{BC} 7$ \\
\hline Untreated & A 104 a & A 140 a & $A 17 b$ & - & Untreated & 69 & 49 & 9 & A 42 \\
\hline Mean & - & - & - & & & $20 a$ & $10 \mathrm{~b}$ & $2 b$ & \\
\hline $\begin{array}{l}\text { Coefficient of } \\
\text { Variation (\%) }\end{array}$ & \multicolumn{4}{|c|}{62} & & \multicolumn{4}{|c|}{143} \\
\hline \multicolumn{10}{|c|}{ Season $2015-2016^{d}$} \\
\hline PRE (g ai ha-1) & 1 & 2 & 3 & & POST (g ai or ae $\cdot \mathrm{ha}^{-1}$ ) & 1 & 2 & 3 & \\
\hline $\begin{array}{c}\text { Atrazine }(1,480)+ \\
\text { S-metolachlor }(1,160)\end{array}$ & $B 1 b$ & C7a & $\mathrm{AOb}$ & - & Untreated & 1 & 21 & 0 & B 7 \\
\hline $\begin{array}{c}\text { Atrazine }(1,480)+ \\
\text { S-metolachlor }(1,160)\end{array}$ & B $2 b$ & $\mathrm{C} 20 \mathrm{a}$ & $A 1 b$ & - & Atrazine $(1,500)$ & 6 & 21 & 0 & B 9 \\
\hline $\begin{array}{c}\text { Atrazine }(1,480)+ \\
\text { S-metolachlor }(1,160)\end{array}$ & $\mathrm{B} 2 \mathrm{~b}$ & $\mathrm{C} 8 \mathrm{a}$ & $\mathrm{AOb}$ & - & $\begin{array}{c}\text { Atrazine }(1,500)+\text { glyphosate } \\
(720)\end{array}$ & 2 & 25 & 2 & B 9 \\
\hline $\begin{array}{c}\text { Atrazine }(1,480)+ \\
\text { S-metolachlor }(1,160)\end{array}$ & $B 1 b$ & C9a & $\mathrm{AOb}$ & - & $\begin{array}{l}\text { Atrazine }(1,500)+\text { ammonium- } \\
\text { glufosinate }(500)\end{array}$ & 6 & 14 & 0 & B 7 \\
\hline $\begin{array}{c}\text { Atrazine }(1,480)+ \\
\text { S-metolachlor }(1,160)\end{array}$ & $\mathrm{B} 2 \mathrm{~b}$ & C 12 a & $\mathrm{AOb}$ & - & $\begin{array}{c}\text { Atrazine }(1,500)+\text { nicosulfuron } \\
(32)\end{array}$ & 0 & 14 & 0 & B 5 \\
\hline Untreated & A $51 a$ & $A B 58$ a & $\mathrm{A} 2 \mathrm{~b}$ & - & $\begin{array}{c}\text { Atrazine }(1,500)+\text { glyphosate } \\
(720)\end{array}$ & 16 & 31 & 1 & B 16 \\
\hline Untreated & A 50 a & A $67 a$ & $\mathrm{~A} 2 \mathrm{~b}$ & - & $\begin{array}{c}\text { Atrazine }(1,500)+\text { ammonium- } \\
\text { glufosinate }(500)\end{array}$ & 7 & 36 & 0 & B 14 \\
\hline Untreated & A $41 a$ & $A B 42 a$ & $\mathrm{~A} 2 \mathrm{~b}$ & - & $\begin{array}{c}\text { Atrazine }(1,500)+\text { nicosulfuron } \\
(32)\end{array}$ & 2 & 25 & 0 & B 9 \\
\hline Untreated & A 39 a & AB 34 a & $A 1 b$ & - & Untreated & 36 & 63 & 2 & A 34 \\
\hline Mean & - & - & - & & & $8 b$ & $27 a$ & $1 \mathrm{c}$ & \\
\hline $\begin{array}{l}\text { Coefficient of } \\
\text { variation (\%) }\end{array}$ & & & & & & & & & \\
\hline
\end{tabular}

Means followed by different lowercase letters in the line are significantly different among sowing date s by Tukey's HSD test ( $p \leq 0.05$ ). Means preceded by different uppercase letters in the column are significantly different among herbicides by Tukey's HSD test ( $\mathrm{p} \leq 0.05)$. ${ }^{\mathrm{a}} \mathrm{Abbreviations:} \mathrm{PRE,} \mathrm{treatment} \mathrm{performed} \mathrm{in} \mathrm{corn}$

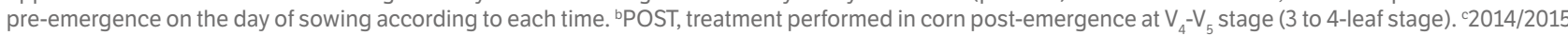
cropping season sowing date: 1) Aug. 22; 2) Sep. 9; 3) Oct. 1. '2015/2016 cropping season sowing date: 1) Sep. 23; 2) Oct. 13; 3) Oct. 28. 
Table 4. The density of Urochloa plantaginea (URPLA) (plants $\mathrm{m}^{-2}$ ) at 28 days after treatment with preemergent herbicides (28 DAPRE) and 21 days after treatment with post-emergent herbicides (21 DAPOST) evaluated in three sowing date s and two cropping seasons $2014 / 2015$ and 2015/2016.

\begin{tabular}{|c|c|c|c|c|c|c|c|c|c|}
\hline \multicolumn{10}{|c|}{ Season $2014-2015^{c}$} \\
\hline \multirow{3}{*}{$\begin{array}{c}\text { Treatments } \\
\left.\text { PRE (g ai } \cdot \mathrm{ha}^{-1}\right)\end{array}$} & \multicolumn{4}{|c|}{ Plants $\mathrm{m}^{-2}$ at 28 DAPRE $^{\mathrm{a}}$} & \multirow{3}{*}{$\begin{array}{c}\text { Treatments } \\
\text { POST (g ai or ae } \cdot \mathrm{ha}^{-1} \text { ) }\end{array}$} & \multicolumn{4}{|c|}{ Plants $\mathrm{m}^{-2}$ at 21 DAPOST $^{\mathrm{b}}$} \\
\hline & \multicolumn{3}{|c|}{ Sowing time } & \multirow[t]{2}{*}{ Mean } & & \multicolumn{3}{|c|}{ Sowing time } & \multirow[t]{2}{*}{ Mean } \\
\hline & 1 & 2 & 3 & & & 1 & 2 & 3 & \\
\hline $\begin{array}{c}\text { Atrazine }(1,480)+ \\
\text { S-metolachlor }(1,160)\end{array}$ & 9 & 3 & 14 & B 9 & Untreated & 45 & 23 & 18 & B 28 \\
\hline $\begin{array}{c}\text { Atrazine }(1,480)+ \\
\text { S-metolachlor }(1,160)\end{array}$ & 12 & 1 & 12 & B 8 & Atrazine $(1,500)$ & 18 & 6 & 9 & C 11 \\
\hline $\begin{array}{c}\text { Atrazine }(1,480)+ \\
\text { S-metolachlor }(1,160)\end{array}$ & 11 & 4 & 14 & B 9 & $\begin{array}{l}\text { Atrazine }(1,500)+ \\
\text { glyphosate }(720)\end{array}$ & 11 & 8 & 5 & C 8 \\
\hline $\begin{array}{c}\text { Atrazine }(1,480)+ \\
\text { S-metolachlor }(1,160)\end{array}$ & 13 & 2 & 16 & B 10 & $\begin{array}{c}\text { Atrazine }(1,500)+\text { ammonium- } \\
\text { glufosinate }(500)\end{array}$ & 13 & 9 & 6 & C9 \\
\hline $\begin{array}{c}\text { Atrazine }(1,480)+ \\
\text { S-metolachlor }(1,160)\end{array}$ & 8 & 4 & 14 & B 9 & $\begin{array}{l}\text { Atrazine }(1,500)+ \\
\text { nicosulfuron }(32)\end{array}$ & 15 & 10 & 7 & C 10 \\
\hline Untreated & 17 & 11 & 37 & A 22 & $\begin{array}{l}\text { Atrazine }(1,500)+ \\
\text { glyphosate }(720)\end{array}$ & 20 & 12 & 4 & C 12 \\
\hline Untreated & 17 & 15 & 56 & A 29 & $\begin{array}{c}\text { Atrazine }(1,500)+\text { ammonium- } \\
\text { glufosinate }(500)\end{array}$ & 19 & 11 & 6 & C 12 \\
\hline Untreated & 17 & 10 & 48 & A 25 & $\begin{array}{l}\text { Atrazine }(1,500)+ \\
\text { nicosulfuron }(32)\end{array}$ & 25 & 12 & 9 & C 15 \\
\hline Untreated & 17 & 12 & 52 & A 27 & Untreated & 85 & 44 & 46 & A 58 \\
\hline Mean & $13 b$ & $7 b$ & $29 a$ & & & $28 a$ & $15 \mathrm{~b}$ & $12 \mathrm{~b}$ & \\
\hline $\begin{array}{l}\text { Coefficient of } \\
\text { Variation (\%) }\end{array}$ & \multicolumn{4}{|c|}{82} & & \multicolumn{4}{|c|}{89} \\
\hline \multicolumn{10}{|c|}{ Season $2015-2016^{d}$} \\
\hline PRE (g ai ha-1) & 1 & 2 & 3 & & POST (g ai or ae $\cdot \mathrm{ha}^{-1}$ ) & 1 & 2 & 3 & \\
\hline $\begin{array}{c}\text { Atrazine }(1,480)+ \\
\text { S-metolachlor }(1,160)\end{array}$ & 10 & 3 & 3 & C5 & Untreated & 13 & 6 & 2 & NS 7 \\
\hline $\begin{array}{c}\text { Atrazine }(1,480)+ \\
\text { S-metolachlor }(1,160)\end{array}$ & 8 & 4 & 4 & C5 & Atrazine $(1,500)$ & 14 & 9 & 4 & 9 \\
\hline $\begin{array}{c}\text { Atrazine }(1,480)+ \\
\text { S-metolachlor }(1,160)\end{array}$ & 9 & 4 & 3 & C5 & $\begin{array}{l}\text { Atrazine }(1,500)+ \\
\text { glyphosate }(720)\end{array}$ & 6 & 8 & 1 & 5 \\
\hline $\begin{array}{c}\text { Atrazine }(1,480)+ \\
\text { S-metolachlor }(1,160)\end{array}$ & 8 & 1 & 5 & $\mathrm{C} 4$ & $\begin{array}{c}\text { Atrazine }(1,500)+\text { ammonium- } \\
\text { glufosinate }(500)\end{array}$ & 9 & 1 & 0 & 3 \\
\hline $\begin{array}{c}\text { Atrazine }(1,480)+ \\
\text { S-metolachlor }(1,160)\end{array}$ & 13 & 3 & 2 & C 6 & $\begin{array}{l}\text { Atrazine }(1,500)+ \\
\text { nicosulfuron }(32)\end{array}$ & 10 & 5 & 0 & 5 \\
\hline Untreated & 23 & 8 & 7 & AB 12 & $\begin{array}{l}\text { Atrazine }(1,500)+ \\
\text { glyphosate }(720)\end{array}$ & 11 & 4 & 2 & 6 \\
\hline Untreated & 17 & 3 & 4 & $A B C 8$ & $\begin{array}{l}\text { Atrazine }(1,500)+\text { ammonium- } \\
\text { glufosinate }(500)\end{array}$ & 13 & 2 & 0 & 5 \\
\hline Untreated & 16 & 4 & 12 & AB 11 & $\begin{array}{l}\text { Atrazine }(1,500)+ \\
\text { nicosulfuron }(32)\end{array}$ & 9 & 4 & 2 & 5 \\
\hline Untreated & 27 & 5 & 10 & A 14 & Untreated & 16 & 4 & 5 & 8 \\
\hline Mean & $14 a$ & $4 b$ & $5 b$ & & & $11 \mathrm{a}$ & $5 b$ & $1.8 \mathrm{c}$ & \\
\hline $\begin{array}{l}\text { Coefficient of } \\
\text { variation (\%) }\end{array}$ & & & & & & & & 4 & \\
\hline
\end{tabular}

Means followed by different lowercase letters in the line are significantly different among sowing date s by Tukey's HSD test ( $p \leq 0.05$ ). Means preceded by different uppercase letters in the column are significantly different among herbicides by Tukey's HSD test ( $\mathrm{p} \leq 0.05)$. ${ }^{\mathrm{a}} \mathrm{Abbreviations:} \mathrm{PRE,} \mathrm{treatment} \mathrm{performed} \mathrm{in} \mathrm{corn}$ pre-emergence on the day of sowing according to each time. ${ }^{b} P O S T$, treatment performed in corn post-emergence at $V_{4}-V_{5}$ stage ( 3 to 4 -leaf stage). ${ }^{c} 2014 / 2015$ cropping season sowing date: 1) Aug. 22; 2) Sep. 9; 3) Oct. 1. '2015/2016 cropping season Sowing date: 1) Sep. 23; 2) Oct. 13; 3) Oct. 28. 
Table 5. Corn grain yield (ton $\mathrm{ha}^{-1}$ ) in response to herbicide treatment in three sowing dates and two cropping seasons 2014/2015 and 2015/2016.

\begin{tabular}{|c|c|c|c|c|c|c|c|c|c|}
\hline \multirow{2}{*}{\multicolumn{2}{|c|}{ Treatments }} & \multicolumn{4}{|c|}{ Cropping Season 2014/2015 } & \multicolumn{4}{|c|}{ Cropping Season $2015 / 2016^{d}$} \\
\hline & & \multicolumn{3}{|c|}{ Sowing date } & \multirow[t]{2}{*}{ Mean } & \multicolumn{3}{|c|}{ Sowing date } & \multirow[t]{2}{*}{ Mean } \\
\hline $\operatorname{PRE}^{\mathrm{a}}\left(\mathrm{g}\right.$ ai $\left.\cdot h a^{-1}\right)$ & $\mathrm{POST}^{\mathrm{b}}$ (g ai or ae $\cdot \mathrm{ha}^{-1}$ ) & 1 & 2 & 3 & & 1 & 2 & 3 & \\
\hline $\begin{array}{c}\text { Atrazine }(1,480)+ \\
\text { S-metolachlor }(1,160)\end{array}$ & Untreated & 13.6 & 13.7 & 16.7 & A 14.7 & 11.3 & 10.9 & 9.9 & B 10.7 \\
\hline $\begin{array}{c}\text { Atrazine }(1,480)+ \\
\text { S-metolachlor }(1,160)\end{array}$ & Atrazine $(1,500)$ & 13.8 & 14.3 & 15.9 & A 14.7 & 11.6 & 12.1 & 10.2 & AB 11.3 \\
\hline $\begin{array}{c}\text { Atrazine }(1,480)+ \\
\text { S-metolachlor }(1,160)\end{array}$ & $\begin{array}{l}\text { Atrazine }(1,500)+ \\
\text { glyphosate }(720)\end{array}$ & 15.4 & 14.2 & 16.4 & A 15.3 & 12.1 & 12.1 & 10.8 & A 11.7 \\
\hline $\begin{array}{c}\text { Atrazine }(1,480)+ \\
\text { S-metolachlor }(1,160)\end{array}$ & $\begin{array}{c}\text { Atrazine }(1,500)+ \\
\text { ammonium-glufosinate }(500)\end{array}$ & 14.7 & 14.7 & 16.6 & A 15.3 & 12.2 & 11.9 & 11.0 & A 11.7 \\
\hline $\begin{array}{c}\text { Atrazine }(1,480)+ \\
\text { S-metolachlor }(1,160)\end{array}$ & $\begin{array}{l}\text { Atrazine }(1,500)+ \\
\text { nicosulfuron }(32)\end{array}$ & 14.1 & 14.6 & 16.0 & A 14.9 & 12.1 & 11.9 & 10.8 & A 11.6 \\
\hline Untreated & $\begin{array}{l}\text { Atrazine }(1,500)+ \\
\text { glyphosate }(720)\end{array}$ & 13.4 & 13.5 & 15.9 & A 14.2 & 10.9 & 12.3 & 10.9 & AB 11.4 \\
\hline Untreated & $\begin{array}{c}\text { Atrazine }(1,500)+ \\
\text { ammonium-glufosinate }(500)\end{array}$ & 14.8 & 14.2 & 16.0 & A 15.0 & 11.3 & 12.2 & 10.2 & AB 11.2 \\
\hline Untreated & $\begin{array}{c}\text { Atrazine }(1,500)+ \\
\text { nicosulfuron }(32)\end{array}$ & 14.7 & 14.5 & 15.1 & A 14.8 & 11.9 & 12.0 & 10.4 & AB 11.4 \\
\hline Untreated & Untreated & 11.7 & 11.1 & 14.7 & B 12.5 & 9.8 & 9.5 & 9.5 & C 9.6 \\
\hline \multicolumn{2}{|c|}{ Mean } & $14.0 \mathrm{~b}$ & $13.9 \mathrm{~b}$ & $15.9 \mathrm{a}$ & & $11.5 \mathrm{a}$ & $11.6 a$ & $10.4 b$ & \\
\hline \multicolumn{2}{|c|}{ Coefficient of variation (\%) } & \multicolumn{4}{|c|}{8.4} & \multicolumn{4}{|c|}{6} \\
\hline
\end{tabular}

Means preceded by different uppercase letters in the column and lowercase letters in line are significantly different among herbicides and sowing dates by Tukey's

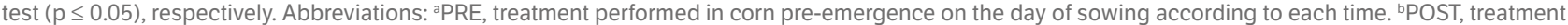
performed in corn post-emergence at $V_{4}-V_{5}$ stage (3 to 4-leaf stage). ${ }^{2} 2014 / 2015$ cropping season Sowing date: 1) Aug. 22; 2) Sep. 9; 3 ) Oct. 1. ${ }^{d} 2015 / 2016$ cropping season sowing date: 1) Sep. 23; 2) Oct. 13;3) Oct. 28.

The results of the present study indicate that corn sown from late August to mid-October was subject to the highest densities of LOLMU, while URPLA showed the most elevated densities when corn was planted from late September to early October. Also, the highest densities of both LOLMU and URPLA coincided in a period from late September to early October. Therefore, the results of the present study provide evidence that the corn sowing date influences the critical period of weed control in corn, being in accordance with another similar study with different weed species (Williams 2006).

\section{Effects of residual herbicide on LOLMU and URPLA densities}

The application of atrazine + S-metolachlor at corn pre-emergence reduced significatively the LOLMU and URPLA densities in both growing seasons (Tables 3 and 4). By comparing the average density of LOLMU and URPLA from those treatments that received preemergent herbicides treatment to the untreated, it is possible to observe a substantial reduction in weed density in both growing seasons regardless of the planting dates (Tables 3 and 4).

The efficiency of the LOLMU control by the atrazine + S-metolachlor in pre-emergence can be observed when it was not followed by post-emergence herbicide treatment. In this case, the LOLMU density did not differ from others treated with pre- and post-emergent or only post-emergent in both growing seasons (Table 3). In the URPLA case, during the first studied growing season, the treatment of only atrazine + S-metolachlor in corn pre-emergence resulted in better control than the untreated, but inferior to those with pre- followed by post-emergence herbicides or only post-emergence. In contrast, during the second season, there was no difference among treatments (Table 4).

The critical period for weed control in corn ranges from $V_{2}$ to $V_{7}$ leaf stage, and the weed interference during the early crop stage causes higher yield losses that can reach up to 87\% (Kozlowski 2002; Page et al. 2012). Also, the time-window from burndown to post-emergence herbicide application may take about 30-45 days. During this period, new flushes of 
LOLMU and URPLA can germinate, grown-up, and reach advanced development stages, which will decrease the likelihood of efficient chemical control. Thus, these results demonstrate that residual herbicides applied in corn pre-emergence are an excellent strategy to control GR LOLMU and URPLA in the early development stages (Tables 3 and 4).

Atrazine is a corn selective PSII inhibitor herbicide with a broad spectrum of control (Philbrook et al. 1999)4. It has widespread use in corn pre- and post-emergence for control of broadleaf, as well as certain grass weeds (Senseman 2007). S-metolachlor is a chloroacetamide herbicide that inhibits very-long-chain fatty acids (VLCFAs), impairing cell division, and shoot development in susceptible plants (Senseman 2007). In annual grasses, S-metolachlor is absorbed by shoot tissues as they grow through the treated soil (LeBaron et al. 1988; Zemolin et al. 2014). Therefore, atrazine + S-metolachlor provided control of LOLMU and URPLA in the early stages of development and contributed to reducing their densities in corn post-emergence. Also, there are no reports related to S-metolachlor resistance to LOLMU and URPLA in Brazil (Heap 2020), and it can be useful for herbicide resistance management.

\section{Effects of post-emergence herbicides on LOLMU and URPLA densities}

The application of all treatment herbicides on corn post-emergence, atrazine, atrazine + glyphosate, atrazine + ammoniumglufosinate, and atrazine + nicosulfuron reduced the LOLMU and URPLA average densities significantly when compared to untreated (Tables 3 and 4). An exception happened during the 2014/2015 season, where the LOLMU average density did not differ from untreated (control) after treatment with atrazine + glyphosate in the post- without preemergent application (Table 3). This result indicates that atrazine alone is not very efficient in controlling LOLMU in post-emergence, and, as expected, the efficiency of glyphosate was low due to resistance. However, when the preemergent herbicides were applied, the control of atrazine + glyphosate was comparable to the other treatments (Tables 3 and 4).

The use of residual herbicides reduced the initial LOLMU and URPLA densities favoring their control in post-emergence. In a recent article, the authors concluded that it is more challenging to control GR LOLMU in high densities and that to reach the same level of control as those obtained with low weed density is necessary to increase the herbicide dose (Fipke and Vidal 2019). However, the results of the present study indicate that the application of atrazine + S-metolachlor in corn pre-emergence is a more efficient alternative than increasing the glyphosate rate or even the number of applications in postemergence. It is because atrazine + S-metolachlor combine two herbicides' mode of action (PSII and VLCFAs), are applied and act when the weeds are at the initial development stages, meaning that it is easier to control them in the early stages (Philbrook et al. 1999; Senseman 2007). Therefore, the combination of the pre- and post-emergent herbicides is a better strategy to be included in the weed management programs than only control in post-emergence. Moreover, the combination of pre- and post-emergence strategies uses diverse modes of action and contributes to the herbicide resistance evolution.

\section{Corn grain yield}

The corn sowing date interfered with the corn yield. During the 2014/2015 crop season, the corn sown on the latest date (Oct 1st) had the highest yield. In the 2015/2016 season, the two earliest sowing dates (Sep 23th and Oct 13th) presented the best corn yield (Table 5). In treatments that received herbicide applications, corn yield was on average 16\% higher than untreated in the 2014/2015 season. In general, during the 2015/2016 season, the best corn yield occurred when the application of herbicides occurred in pre- followed by post-emergence, only in post-emergence, only in pre-emergence, and untreated, respectively in order of importance (Table 5).

Despite the occurrence of LOLMU and URPLA in various densities, corn yield was not drastically reduced by weed interference, even when the herbicide treatment was performed only in pre- or only in post-emergence (Table 5). Corn yield is dependent of several factors, such as water, nutrients, and radiation (Radosevich et al. 2007). The insignificant effects of

4. Philbrook, B. D., Kremer, M., Mueller, K. H. and Deege, R. (1999). BAY MKH 3586 - a new herbicide for broad spectrum weed control in corn (maize) and sugar cane. In 1999 Brighton crop protection conference: weeds Proceedings of an international conference. Brighton. [Accessed June 06 2019]. Available at: https://eurekamag.com/research/003/367/003367383.php 
weed interference observed on corn yield might be attributed to not any resource limitation for the crop during a significant part of the development, particularly during the critical period of weed interference (Kozlowski 2002; Page et al. 2012). Also, the corn was cultivated in a narrow space of $0.5 \mathrm{~m}$ between rows favoring crop development and its fast occupation of the area, known effect usually described as cultural control (Radosevich et al. 2007). Moreover, the onset and severity of the weed interference on crop yield losses vary across fields and years, often with a nonsignificant connection to the levels of weed interference or timing of weed management actions (Evans et al. 2003; Hall et al. 1992).

Finally, combining all the findings of the present study related to sowing date, residual preemergent and post-emergent herbicides, the results provide evidence that those treatments influence on the critical period of weed control. Therefore, the adoption of cultural and chemical strategies on the management of LOLMU and URPLA is efficient. Likewise, combined-strategies accumulate positive benefits from different weed management approaches, being useful to herbicide resistance management.

\title{
CONCLUSION
}

The results of the present study indicate that the corn sowing date interferes with the density of LOLMU and URPLA during the corn growth cycle. In this case, when corn is sown in the earliest periods (August to September), it will be expected higher density of LOLMU, whereas, in the latest sowing dates (November), it will likely be observed more URPLA plants interfering with the crop. In addition, the application of residual herbicides at corn pre-emergence reduced the LOLMU and URPLA densities favoring the control in post-emergence by glyphosate, ammonium-glufosinate, atrazine, and nicosulfuron.

\section{ACKNOWLEDGMENTS}

\author{
Conselho Nacional de Desenvolvimento Científico e Tecnológico (CNPq) \\ [http://doi.org/10.13039/501100003593]
}

Coordenação de Aperfeiçoamento de Pessoal de Nível Superior

[http://doi.org/10.13039/501100002322]

Convênio Embrapa/Bayer pelo financimaneto da pesquisa

\section{AUTHOR'S CONTRIBUTION}

Conceptualization, R. F. L. Ovejero, G. J. Picoli Junior and L. F. Bellini; Methodology, R. F. L. Ovejero, G. J. Picoli Junior, and L. F. Bellini; Investigation, R. F. L. Ovejero, G. J. Picoli Junior, L. F. Bellini., C. Piasecki, L. Vargas and D. Agostinetto; Data Curation, C. Piasecki, L. F. Bellini, R. F. L. Ovejero.; Formal Analysis, C. Piasecki; Funding Acquisition, R. F. L. Ovejero, G. J. Picoli Junior, L. F. Bellini, D. Agostinetto and L. Vargas; Project Administration, R. F. L. Ovejero, G. J. Picoli Junior, D. Agostinetto and L. Vargas; Software, C. Piasecki; Supervision, R. F. L. Ovejero, G. J. Picoli Junior, and L. Vargas; Writing - Original Draft, C. Piasecki; Writing - Review and Editing, C. Piasecki, R. F. L. Ovejero, G. J. Picoli Junior, L. F. Bellini, L. Vargas, and D. Agostinetto.

\section{REFERENCES}

[CONAB] Companhia Nacional de Abastecimento. (2018). Acompanhamento da safra brasileira de grãos: X levantamento $2017 / 2018$. Brasília: CONAB. [Accessed Mar. 26, 2018]. Available at: https://www.conab.gov.br/info-agro/safras/graos 
[EMBRAPA] Empresa Brasileira de Pesquisa Agropecuária. (1999). Centro Nacional de Pesquisa Agropecuária de Solos. Sistema brasileiro de classificação de solos. Brasília: Embrapa Produção de Informação; Rio de Janeiro: Embrapa Solos.

Banzatto, D. A. and Kronka, S. N. (2008). Experimentação agrícola. Jaboticabal: Funep.

Bond, J. A., Eubank, T. W., Bond, R. C., Golden, B. R. and Edwards, H. M. (2014). Glyphosate-resistant Italian ryegrass (Lolium perenne ssp. multiflorum) control with fall-applied residual herbicides. Weed Technology, 28, 361-370. https://doi.org/10.1614/WT-D-13-00149.1

Cardinali, A. and Nason, G. P. (2013). Costationarity of locally stationary time series using costat. Journal of Statistical Software, 55. https://doi.org/10.18637/jss.v055.i01

Evans, S. P., Knezevic, S. Z., Lindquist, J. L., Shapiro, C. A. and Blankenship, E. E. (2003). Nitrogen application influences the critical period for weed control in corn. Weed Science, 51, 408-417. https://doi.org/10.1614/0043-1745(2003)051[0408:NAITCP]2.0.CO;2

Fipke, M. V. and Vidal, R. A. (2019). Influence of density and development stage of ryegrass on glyphosate effectiveness. Planta Daninha, 37, e019178173. https://doi.org/10.1590/s0100-83582019370100025

Galon, L., Pinto, J. J. O., Rocha, A. A., Concenço, G., Silva, A. F., Aspiazú, I., Ferreira, E. A., França, A. C., Ferreira, F. A., Agostinetto, D. and Pinho, C. F. (2008). Períodos de interferência de Brachiaria plantaginea na cultura do milho na região Sul do Rio Grande do Sul. Planta Daninha, 26, 779-788. https://doi.org/10.1590/S0100-83582008000400009

Gramshaw, D. (1972). Germination of annual ryegrass seeds (Lolium rigidum Gaud.) as influenced by temperature, light, storage environment and age. Australian Journal of Agricultural Research, 23, 779-787. https://doi.org/10.1071/AR9720779

Hall, M. R., Swanton, C. J. and Anderson, G. W. (1992). The critical period of weed control in grain corn (Zea mays). Weed Science, 40, 441-447. https://doi.org/10.1017/S0043174500051882

Heap, I. (2020). The International Survey of Herbicide Resistant Weeds. [Accessed Mar. 21, 2020]. Available at: http://www.weedscience. org/Home.aspx

Khatounian, C. A., Passini, T., Penha, L. A. O. and Oliveira, D. A. M. (2016). Seed production of Urochloa plantaginea (Link) R. Webster infesting maize and in pure stands. Revista Brasileira de Agroecologia, 11, 281-288.

Kozlowski, L. A. (2002). Período crítico de interferência das plantas daninhas na cultura do milho baseado na fenologia da cultura. Planta Daninha, 20, 365-372. https://doi.org/10.1590/S0100-83582002000300006

LeBaron, H. M., McFarland, J. E., Simoneaux, B. J. and Ebert, E. (1988). Metolachlor. In P. C. Kearney and D. D. and Kaufman (Eds.), Herbicides: chemistry, degradation and mode of action (p. 335-373). NewYork: Marcel Dekker.

Macedo, L. C. P., Dornelles, S. H. B., Peripolli, M., Trivisiol, V. S., Conceição, D. Q., Pivetta, M., Escobar, O. S. and Essi, L. (2019). Phenology and dry mass production of Urochloa plantaginea and Urochloa platyphylla submitted to different water quantities in the soil. Acta Scientiarum. Biological Sciences, 41, e46127.

Nandula, V. (2014). Italian Ryegrass (Lolium perenne ssp. multiflorum) and Corn (Zea mays) Competition. American Journal of Plant Sciences, 5, 3914-3924. https://doi.org/10.4236/ajps.2014.526410

Page, E. R., Cerrudo, D., Westra, P., Loux, M., Smith, K., Foresman, C., Wright, H. and Swanton, C. J. (2012). Why early season weed control is important in maize. Weed Science, 60, 423-430. https://doi.org/10.1614/WS-D-11-00183.1

Radosevich, S. R., Holt, J. S. and Ghersa, C. M. (2007). Ecology of weeds and invasive plants: relationship to agriculture and natural resource management. Hoboken: John Wiley \& Sons. https://doi.org/10.1002/9780470168943

Roman, E. S., Vargas, L., Rizzardi, M. A. and Mattei, R. W. (2004). Resistência de azevém (Lolium multiflorum) ao herbicida glyphosate. Planta Daninha, 22, 301-306. https://doi.org/10.1590/S0100-83582004000200018

Senseman, S. A. (2007). Herbicide handbook. Lawrence: Weed Science Society of America. 
Vargas, L., Adegas, F., Gazziero, D., Karam, D., Agostinetto, D. and Silva, W. T. (2016). Resistência de plantas daninhas a herbicidas no Brasil: histórico, distribuição, impacto econômico, manejo e prevenção. In D. K. Meschede and D. L. P. Gazziero (Eds.), A era glyphosate: agricultura, meio ambiente e homem (p. 219-239). Londrina: Midiograf II.

Vargas, L., Roman, E. S., Rizzardi, M. A. and Silva, V. C. (2005). Alteração das características biológicas dos biótipos de azevém (Lolium multiflorum) ocasionada pela resistência ao herbicida glyphosate. Planta Daninha, 23, 153-160. https://doi.org/10.1590/ S0100-83582005000100018

Velho, G. F. Crusciol, C. A. C., Velini, E. D., Castro, G. S. A. and Borghi, E. (2012). Interferência de Brachiaria plantaginea com a cultura do arroz, cv. Primavera. Planta Daninha, 30, 17-26. https://doi.org/10.1590/S0100-83582012000100003

Williams, M. M. (2006). Planting date influences critical period of weed control in sweet corn. Weed Science, 54, 928-933. https://doi. org/10.1614/WS-06-005R.1

Zemolin, C. R., Avila, L. A., Cassol, G. V., Massey, J. H. and Camargo, E. R. (2014). Environmental fate of s-metolachlor - a review. Planta Daninha, 32, 655-664. https://doi.org/10.1590/S0100-83582014000300022 